\title{
Autologous platelet gel and fibrin sealant enhance the efficacy of total knee arthroplasty: improved range of motion, decreased length of stay and a reduced incidence of arthrofibrosis
}

\author{
Peter A. M. Everts · Roger J. J. Devilee • Cornelis J. M. Oosterbos • \\ Christine Brown Mahoney • Maarten Eeftinck Schattenkerk • \\ Johannes T. A. Knape · André van Zundert
}

Received: 17 August 2006/ Accepted: 22 January 2007/Published online: 24 February 2007

(C) Springer-Verlag 2007

\begin{abstract}
In this study we describe the potential role of autologous platelet gel and fibrin sealant in unilateral total knee arthroplasty to improve the postoperative range of motion and to reduce the incidence of arthrofibrosis. Total knee arthroplasty is often associated with a considerable amount of post-operative blood loss. Persistent limited motion directly after surgery may ultimately result in arthrofibrosis. To counteract these effects we investigated whether the use of autologous derived platelet gel and fibrin sealant would reduce postoperative blood loss, decrease the impaired range of motion and the incidence of arthrofibrosis. All patients were consecutively operated and assigned to the study or control groups. Study
\end{abstract}

\section{P. A. M. Everts}

Department of Peri-Operative Blood Management,

Catharina Hospital, Eindhoven, The Netherlands

R. J. J. Devilee · C. J. M. Oosterbos - M. E. Schattenkerk

Department of Orthopaedic Surgery-Traumatology,

Catharina Hospital, Eindhoven, The Netherlands

C. B. Mahoney

Business Schools of Management and Statistics,

Hamline University, St Paul, MN, USA

J. T. A. Knape

Department of Anesthesiology, University Medical Centre, Utrecht, The Netherlands

A. van Zundert

Department of Anesthesiology, Catharina Hospital,

Eindhoven, The Netherlands

P. A. M. Everts $(\square)$

Department of ECC/BM, Catharina Hospital,

Michelangelolaan 2, 5623 EJ Eindhoven, The Netherlands

e-mail: everts@elive.nl group patients $(n=85)$ were treated with the application of autologous platelet gel and fibrin sealant at the end of surgery. Eighty patients were operated without the use of platelet gel and fibrin sealant, and served as the control group. The postoperative hemoglobin decrease, range of motion and length of hospitalization were recorded. During a 5-month postoperative period patients were followed to observe the incidence of arthrofibrosis. In patients in the treatment group the hemoglobin concentration in blood decreased significantly less when compared to the control group. They also showed a superior postoperative range of motion when compared to those of the control group $(P<0.001)$. The incidence of arthrofibrosis and subsequent forced manipulation was significantly less $(P<0.001)$ in patients managed with platelet gel and fibrin sealant. We conclude that peri-operatively applied platelet gel and fibrin sealant may improve the range of motion after total knee arthroplasty, decreases the length of stay and may reduce the incidence of arthrofibrosis.

Keywords Total knee arthroplasty · Platelet gel · Fibrin sealant $\cdot$ Range of motion - Arthrofibrosis

\section{Introduction}

Total knee arthroplasty is often associated with a considerable amount of postoperative blood loss, necessitating the transfusion of allogeneic blood products [3]. Relentless postoperative bleeding can contribute to impaired outcomes after total knee arthroplasty, e.g., due to the development of wound haematoma and seroma formation. Control of haemostasis following 
total knee arthroplasty is an important concern since bleeding might lead to the formation of adhesions and might even cause arthrofibrosis [6, 41]. Arthrofibrosis is rare, although its incidence may be up to $10 \%$ $[11,17]$.

Optimization of strategies to improve outcome after total knee arthroplasty aims to reduce postoperative bleeding, to improve the range of motion and to decrease the incidence of arthrofibrosis. Platelet gel (PG), a so-called buffy coat product prepared from freshly drawn autologous blood, is a mixture of platelet and leukocyte rich plasma being activated with thrombin to produce a viscous gel cloth [8]. PG delivers concentrated platelets and the platelet growth factors directly to wound sites, specifically plateletderived growth factor and transforming growth factor$\beta$, which both could contribute to improve wound healing $[5,20]$.

Treatment with autologous, bed-side prepared blood components, in order to control bleeding and stimulate wound healing, is a relatively new concept and might reduce the development of tissue adhesions. Fibrin sealants prepared from platelet poor plasma (PPP) and also activated with thrombin, have been used in a variety of surgical procedures in order to control bleeding, with a subsequent reduction in postoperative tissue adhesions [24, 33, 35].

In orthopaedic surgery, PG has mostly been used in spinal fusion operations to enhance fusion, since platelets stimulate the activity of bone cells, based on their particular relevance to bone growth and therefore accelerate bone growth [34, 37]. Recently, PG has also been used to control bleeding [7, 10]. Fibrin sealants have mainly been used as reconstructive supportive surgical tools [30]. PG and fibrin sealants have also been cited as useful in soft tissue tendon repairs, $[1,2]$ and as a supportive tool in bone regeneration [9, 42].

In this study we assessed the potential of PG and fibrin sealant to act as a haemostatic-surgical tool during total knee arthroplasty and if it might improve the range of motion and probably decrease the incidence of arthrofibrosis up to 5 months after surgery.

\section{Materials and methods}

In this observational study, 165 patients who were scheduled for primary unilateral total knee arthroplasty were included. All patients were consecutively operated and underwent the same surgical procedure performed by the same two surgeons (RD or MES). The indication in all patients for total knee arthroplasty was osteoarthritis of the knee. Eighty-five patients were treated with PG and fibrin sealant and a further 80 patients served as untreated controls. They were all operated under a standardized spinal anesthesia and fluid regimen protocol. A tourniquet was used in all cases. Patients with a preoperative-hemoglobin level less than $12 \mathrm{~g} / \mathrm{dl}$ or know coagulation disorders were excluded from the study.

The autologous blood components were prepared from a unit of freshly drawn whole blood (approximately $350 \mathrm{ml}$ ) using an infusion line, which was been placed in the medial cubital vein into a bag containing anticoagulant to avoid blood clothing. A blood cell separator, (Electa ${ }^{\mathrm{TM}}$, Sorin Group, Mirandola, Italy), sequestered the whole blood in fractions of platelet rich plasma (PRP) and PPP [7]. To collect the PRP and PPP, a single centrifugation procedure with a $125 \mathrm{ml}$ Latham bowl was performed. The centrifugation process consisted of a $5,400 \mathrm{rpm}(1,660 \mathrm{~g}$ force $)$ and a $2,400 \mathrm{rpm}$ cycle (305g force) for 15 and $3 \mathrm{~min}$, respectively.

A medial para-patellar approach was used, averting the patella laterally. With the use of a tourniquet, cemented and non-cemented cruciate-sparing prostheses were implanted (Advantim and Advance, Wright Medical Products, Arlington, TN and Somas, The Netherlands; LCS, Johnson and Johnson DePuy, Piscataway, NJ). After implantation of the prosthesis the tourniquet was deflated, and primary haemostasis of major blood vessels was achieved with electrocautery. Before wound closure, the knee soft tissues and joint were rinsed with saline solution to remove all debris. PG and fibrin sealants were then produced from PRP and PPP, respectively, by the addition of calcified thrombin to set up a PG blood cloth and create a sticky fibrin sealant. Autologous thrombin was produced from PRP by mixing $6 \mathrm{ml}$ of PRP with $0.25 \mathrm{ml}$ of calcium chloride $10 \%$. PG was created by mixing PRP with autologous thrombin at a ratio of $4: 1$. Fibrin sealant was produces by mixing $10 \mathrm{ml}$ PPP with $1 \mathrm{ml}$ autologous thrombin in the aerosol delivery system (Aerosol Ratio Applicator, Micromedics Inc., St Paul, MN, USA).

PG and fibrin sealants were applied to dried wound sites in order to promote adherence of the components to the tissues. In the study group, the knee was positioned in approximately $90^{\circ}$ flexion to expose the knee cavity, femoral and tibial components. Ten millilitre of PG was initially injected in the back of the knee cavity to plug the bone marrow sinusoids (Fig. 1), the posterior recess, the gutters and the raw exposed surfaces of the femur and tibia.

Thereafter, the wound area was retracted and $10 \mathrm{ml}$ of fibrin sealant was applied to the dried tissues by a 


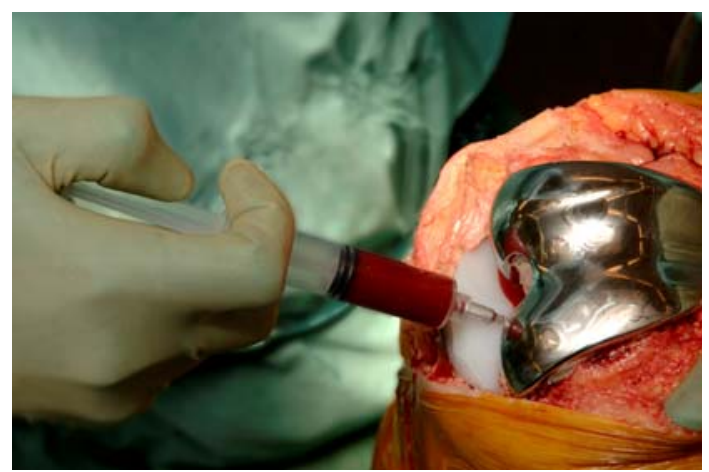

Fig. 1 Injection of platelet gel in the back of the knee cavity after prosthetic implant

double syringe technique and delivered by aerosol topical spraying at a distance of $15 \mathrm{~cm}$ (Fig. 2) to prevent the formation of hematoma and seromata. The wound was closed in layers, with the knee in approximately $90^{\circ}$ flexion. After closure of the knee capsula, another $10 \mathrm{ml}$ of PG was injected between the stitches on the repaired extensor mechanism and the prepatellar fat (Fig. 3). The closed capsula and the subcutaneous tissues were again dried and sprayed with $8 \mathrm{ml}$ of fibrin sealant, and the skin was closed with skin staples.

In all patients, the knee incision was dressed postoperatively with compressing bandages and rehabili-

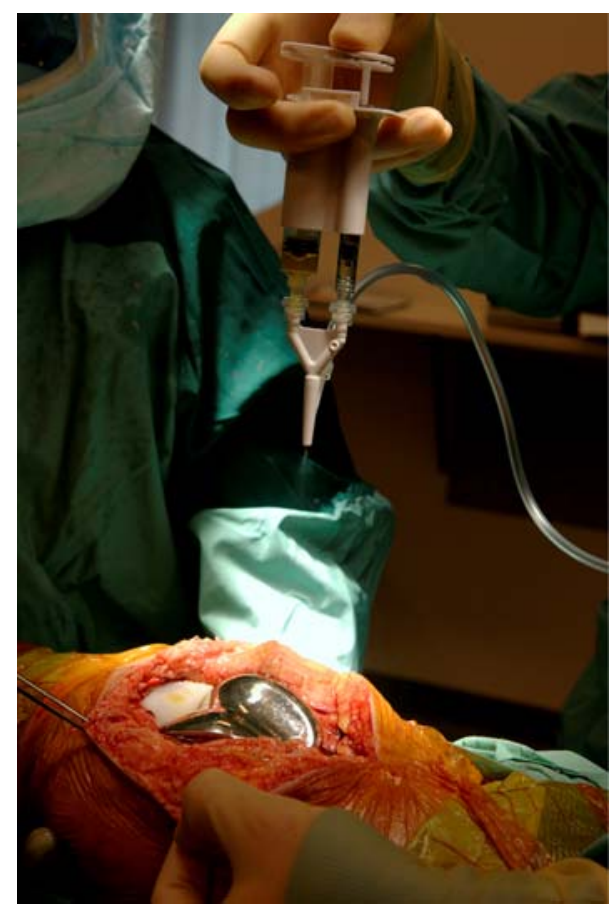

Fig. 2 Application of fibrin sealant to the dried tissues by aerosol topical spraying with a double syringe technique

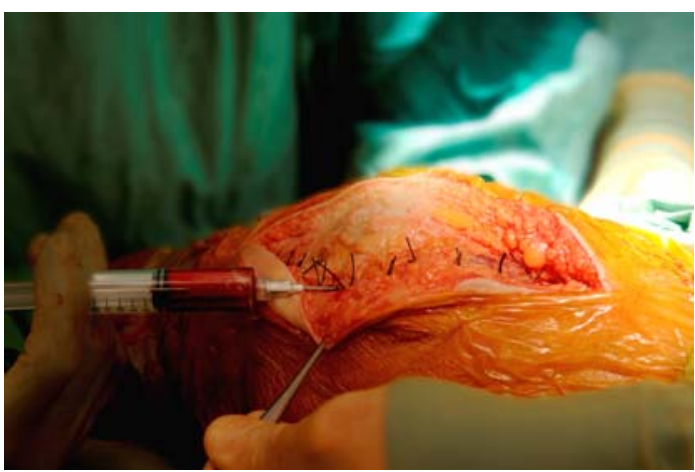

Fig. 3 Injection of platelet gel between the stitches of the repaired extensor mechanism and the pre-patellar fat

tation was started at the day of surgery, with constant passive motion that was increased daily within the patient's pain tolerance. Postoperative pain relief was achieved using a standard protocol, (paracetamol $3 \mathrm{~g}$ daily and naproxen $500 \mathrm{mg}$ twice daily for the first two postoperative days. If necessary, $10-20 \mathrm{mg}$ piritramide was given).

All patients received thrombosis prophylaxis, before the operation until 6 weeks postoperatively, via a subcutaneous injection of $20 \mathrm{mg}$ nadroparin (Fraxiparine ${ }^{\circledR}$ ).

Decreases in postoperative hemoglobin levels, the range of motion (expressed as maximum degrees of flexion of the knee joint) during the first 5 days and at discharge, and the incidence of postoperative arthrofibrosis were all recorded. We defined arthrofibrosis as (painful) stiffness with scarring of soft tissue, a limited flexion $\left(<80^{\circ}\right)$ and patellofemoral immobility, with no heterotopic bone formation. The ward physician or nurse practitioner was blinded for the study during the daily determination of the postoperative range of motion.

Length of hospital stay was recorded in all patients. Patients were discharged if they met all of the following criteria: joint function recovery with a flexion $>90^{\circ}$, absence of wound leakage, no wound healing disturbances and no signs of infection.

Statistical analyses were performed using SAS statistical software (SAS Institute, Cary, NC, USA, 2003). Categorical data are expressed as a percentage with 165 patients included and measured data are expressed as mean \pm standard deviation. A univariate analysis was performed by means of Fisher's exact test on all categorical data and the Student's $t$-test on all measured data. Differences between means were analyzed using the Student's $t$-test and Satterthwaite's approximation that does not assume equal variances [16]. A two-sided $P$ value of 0.05 or less was considered to be statistically significant. 
Table 1 Patient characteristics and type of knee prosthesis used

\begin{tabular}{|c|c|c|}
\hline Description & $\begin{array}{l}\text { Treatment group } \\
(n=85)\end{array}$ & $\begin{array}{l}\text { Control group } \\
(n=80)\end{array}$ \\
\hline Age (years) & $69.4 \pm 9.1$ & $67.4 \pm 9.9$ \\
\hline Gender (F/M) & $58 / 27$ & $58 / 22$ \\
\hline$\%$ Cemented & 74 & 73 \\
\hline $\begin{array}{l}\text { Pre-op hemoglobin (g/ } \\
\text { dl) }\end{array}$ & $13.6 \pm 1.1$ & $13.7 \pm 1.1$ \\
\hline Post-op EC-T (Units) & $0.17 \pm 0.6$ & $0.52 \pm 0.9^{*}$ \\
\hline $\begin{array}{l}\text { Discharge hemoglobin } \\
(\mathrm{g} / \mathrm{dl})\end{array}$ & $10.9 \pm 1.0$ & $10.5 \pm 1.1$ \\
\hline
\end{tabular}

The values are given as mean and the standard deviation $E C$ - $T$ erythrocyte concentrate transfusion

$* P<0.001$. The mean EC-T was significantly higher in the control group

\section{Results}

The mean age of the PG and fibrin sealants treated patients $(n=85)$ and control patients $(n=80)$ were similar $(69.4 \pm 9.1$ and $67.4 \pm 9.9$, respectively). The distribution of gender was $68 \%$ females in the treatment group and $72 \%$ in the control (not statistically different). The distributions for cementless and cemented prosthetic implants were similar for both groups.

The pre-operative hemoglobin value was also comparable in both groups (Table 1). Postoperative hemoglobin values of patients receiving PG and fibrin sealant dropped significantly less in the study group $(P<0.001)$ when compared to the control patients $(2.5$ vs. $5.2 \mathrm{~g} / \mathrm{dl}$, respectively).

Range of motion data are shown in Fig. 4. Functional knee recovery occurred significantly earlier in treated patients. At the fourth postoperative day the functional range of motion was $82.4 \pm 9^{\circ}$ and $66.1 \pm 13^{\circ}$ $(P<0.001)$ for the $\mathrm{PG} /$ fibrin sealant patients and control patients, respectively. By the fifth postoperative day the range of motion was $92.4 \pm 8^{\circ}$ and $79.2 \pm 9^{\circ}$, respectively $(P<0.001)$. Further analysis

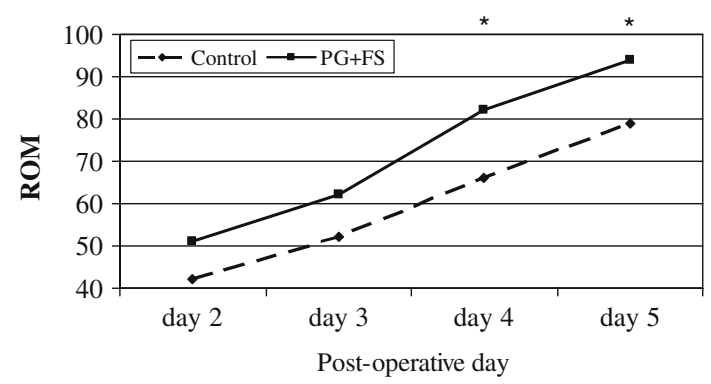

Fig. 4 Development of the range of motion in the postoperative period. $R O M$ range of motion, $P G+F S$ platelet gel and fibrin sealant. $* P<0.001$ revealed that patients with cemented implants and treated with PG/fibrin sealant, showed the same statistical difference when compared to the same patients in whom no PG/fibrin sealant was employed with regard to development in the ROM $(P<0.001)$.

None of the patients underwent TKA for rheumatoid arthritis. In Table 2 the incidence of arthrofibrosis, up to 5 months after surgery is shown. The preoperative active flexion was not statistical different for both patient groups. The criterion for arthrofibrosis was met in all patients (flexion $70^{\circ}$ and $79^{\circ}$ for treated and control patients, respectively). Arthrofibrosis occurred only patients who received a cemented prosthesis. This complication arised only in two patients in whom PG and fibrin sealants were applied, whereas eighth control patients developed arthrofibrosis $(P<0.001)$ (Table 2). After forced mobilization the active flexion improved by 28 and $24 \%$ for treated and control patients, respectively.

The improved range of motion and reduced bleeding contributed to a shorter hospitalization time in treated patients $(6.9 \pm 1.4$ days $)$ compared to the control group $(8.3 \pm 2.9$ days $)(P<0.001)$.

\section{Discussion}

Total knee arthroplasty is associated with major blood loss particularly in the postoperative period following the release of the tourniquet. In this study, we found that in patients treated with the exogenous application of PG and fibrin sealant, the drop in hemoglobin was less.

Various reports from clinical and experimental studies have shown that the use of fibrin sealants to seal off tissue capillaries can be an effective measure to reduce the incidence of bleeding and oozing after surgical interventions. Both experimental and clinical data have demonstrated the efficacy of wound sealing $[25,36]$. However, only few clinical studies with patients undergoing total knee arthroplasty in combination with haemostatic agents to control bleeding have been published. Levi et al. [18] and Wang [40] used commercially available topical sprays, to reduce bleeding. However, the effect of reduced bleeding on the postoperative range of motion and the occurrence of arthrofibrosis was not investigated.

Arthrofibrosis after total knee arthroplasty, which has been variably referred to as stiffness and limited motion, is a disabling complication. There are only a few studies, which show the prevalence of arthrofibrosis to vary from 1 to $11 \%$ [11, 17, 32]. These differences may be explained by differences in the definitions. In the 
Table 2 Knee flexion data in patients with arthrofibrosis

\begin{tabular}{lclcc}
\hline & Pre-op flex & Post-op flex & Weeks after surgery & Post FM flex \\
\hline Control $(n=8)$ & $113 \pm 27$ & $79 \pm 11$ & $14 \pm 6$ & $98 \pm 10$ \\
PG + FS $(n=2)$ & $98 \pm 8$ & $70 \pm 6$ & $8 \pm 3$ & $90 \pm 4$ \\
\hline
\end{tabular}

Data are given in degrees (mean $\pm \mathrm{SD})$

$P G+F S$ platelet gel and fibrin sealant, Pre-op flex preoperatively flexion, Post-op flex postoperatively flexion, Post FM flex postoperative flexion after forced knee mobilization/manipulation under epidural anesthesia at the first visit after the procedure

majority of these studies, arthrofibrosis was defined as a flexion contraction. In this study we used the criteria as defined by Maloney [19]. Others have used slightly different definitions $[4,23]$ for arthrofibrosis or stiffness. Arthrofibrosis may be caused by numerous factors, including impaired range of motion pre-operatively, surgical technical problems, and inadequate postoperative rehabilitation with limiting motion until wound healing occurs or a biological predilection $[11,14,22,26$, 28]. Some patients may be predisposed to extensive scar tissue formation as a response to the tissue trauma, which occurs during the surgical intervention. Nevertheless, in our study we could not detect any underlying factors in either patient group that would increase the risk for the development of arthrofibrosis.

In addition to the prevention of bleeding and oozing, sealing of surgical tissues also may contribute to reduced post-surgical adhesion [13, 39]. Sheppard and associates [33] demonstrated in a long-term model that animals treated with wound sealing had significantly less high-grade adhesions. In addition, sealing of wound tissues might decrease inflammatory processes following surgical interventions, and thus to a reduction of adhesion formation $[12,38]$.

Autologous blood sequestration of whole blood and the preparation of $\mathrm{PG}$ in the peri-operative period is a simple and highly reproducible procedure creating a biological carrier of both the patient's own platelets and leucocytes [15]. PRP is a buffy coat product, containing high concentrations of platelets with at least six platelet growth factors inside the $\alpha$-granules, each having a specific function during wound repair [21]. Upon the creation of a PG, the addition of thrombin activates platelets and they subsequently release their granule contents in a way that various growth factors interact with specific receptor cells and thus initiate the healing process [31]. In addition, due to the activation process more platelets are attracted to the wound site to augment the natural healing processes. PRP also contains a high concentration of non-activated functional neutrophilic white blood cells. These white blood cells contribute to the inhibitory effects of inflammatory processes [27] and may act as antimicrobial agents. Based on the results of applying wound sealants in a variety of surgical settings, we developed in this study a combination of exogenously applied PG and fibrin sealant for total knee arthroplasty. The preparation of the syringes for PG delivery and fibrin sealant use during wound closure were prepared by a person educated and trained in autologous platelet growth factor delivery and fibrin sealant application techniques. This person scrubbed up to the surgical team at the end of the procedure to assist during the application of the components. Worth mentioning is the fact that due to this individual, the total surgical time was hardly increased.

Particular emphasis was to reduce postoperative bleeding, to achieve an earlier increased range of motion after surgery, to minimize soft tissue adhesions and to reduce the development of arthrofibrosis.

Patients treated with PG and fibrin sealant could achieve a significantly greater range of motion when compared to non-treated patients. In another study, a similar increased range of motion in patients treated with PG and fibrin sealants has been reported [10]. As noted in the literature, the preoperative range of motion is a good predictor of the postoperative range of motion $[19,29]$ and is the most important variable in determining the patient's postoperative flexion. A total knee arthroplasty will not improve flexion in patients who have preoperatively a stiff knee. In this study we observed that the postoperative range of motion of the knee was always inferior to the preoperative range of motion. Furthermore, we could not demonstrate an effect of cemented versus cementless prosthesis with regard to the development in the ROM in this study. Therefore, the effect of PG/fibrin sealant application contributed to an improved outcome, regardless the type of prosthesis used.

Another effect by applying PG and fibrin sealant was that the total admission time was shortened by $1.4 \pm .0 .4$ days $(P<0.001)$, when the same criteria for discharge were used for both groups. These data suggest that the application of autologous PG may contribute to better wound tissue healing. Also the coverage of oozing wound sites may minimize adhesion and soft tissue contractions, probably contributing to a lower incidence of arthrofibrosis in the treated 
patients. Patients, in whom arthrofibrosis was diagnosed, were later subjected to forced mobilization under general anesthesia, which resulted in increased mobility of the knee joint in all patients.

In conclusion, we have shown that peri-operative applied PG and fibrin sealant in total knee arthroplasty may contribute to an earlier and increased range of motion of the knee joint and may reduce the incidence of arthrofibrosis associated with total knee arthroplasty. The application of autologous growth factors in orthopaedic surgery could be developed into a routine procedure. Future randomized controlled studies are now warranted to elucidate the potential effect of PG and fibrin sealant in a variety of orthopaedic surgical procedures.

Acknowledgments The authors wish to thank Mr. G. van Dael and M. Roelofs for the preparation of the Figs. 1, 2, and 3.

\section{References}

1. Anitua E, Andia I, Sanchez M et al (2005) Autologous preparations rich in growth factors promote proliferation and induce VEGF and HGF production by human tendon cells in culture. J Orthop Res 23:281-286

2. Aspenberg P, Virchenko O (2004) Platelet concentrate injection improves Achilles tendon repair in rats. Acta Orthop Scand 75:93-99

3. Bierbaum BE, Callaghan JJ, Galante JO, Rubash HE, Tooms RE, Welch RB (1999) An analysis of blood management in patients having a total hip or knee arthroplasty. J Bone Joint Surg Am 81:2-10

4. Christensen CP, Crawford JJ, Olin MD, Vall TP (2002) Revision of stiff total knee arthroplasty. J Arthroplasty 17:409-415

5. Crovetti G, Martinelli G, Issi M et al (2004) Platelet gel for healing cutaneous chronic wounds. Transfus Apher Sci 30:145-151

6. Dennis DA (1997) Wound complications in total knee arthroplasty. Instr Course Lect 46:165-169

7. Everts PAM, Devilee RJJ, Brown-Mahoney C et al (2006) Platelet gel and fibrin sealant reduce allogenic blood transfusions and in total knee arthroplasty. Acta Anaesthesiol Scand 50:593-599

8. Everts PAM, Hoffmann JJHL, Weibrich G et al (2006) Autologous platelet gel growth factor release and leukocyte kinetics using three devices. Transfus Med 16:363-368

9. Gandhi A, Dumas C, O'Conner JP, Parsons JR, Lin SS (2006) The effects of local platelet delivery on diabetic fracture healing. Bone 38:540-546

10. Gardner MJ, Demetrakopoulos D, Klepchick PR, Mooar PA (2006) The efficacy of autologous platelet gel in pain control and blood loss in total knee arthroplasty. Int Orthop. doi: 10.1007/s00264-006-0174-z

11. Gollwitzer H, Burgkart R, Diehl P, Gradinger R, Buhren V (2006) Therapy of arthrofibrosis after total knee arthroplasty. Orthopade 35:143-152

12. Jahoda AE, Albala DM, Dries DJ, Kovacs EJ (1999) Fibrin sealant inhibits connective tissue deposition in a murine model of peritoneal adhesion formation. Surgery 125:53-59

13. Jones ME, Burnett S, Southgate A, Sibbons P, Grobbelaar AO, Green CJ (2002) The role of human-derived fibrin sealant in the reduction of postoperative flexor tendon adhesion formation in rabbits. J Hand Surg (Br) 27:278-282

14. Jordan LR, Olivo JL (1999) Stiffness and limitation of motion; prevention and management. In: Lotke PA, Garino JP (eds) Revision total knee arthroplasty. Lippencott-Raven, Philadelphia, pp 461-467

15. Kevy SV, Jacobson MS (2004) Comparison of methods for point of care preparation of autologous platelet gel. J Extra Corpor Technol 36:28-35

16. Khuri AI, Mathew T, Nel DG (1994) A test to determine closeness of multivariate Satterthwaite's approximation. J Multivariate Anal 51(1):201-209

17. Kim J, Nelson CH, Lotke PA (2004) Stiffness after total knee arthroplasty. J Bone Joint Surg Am 86:1479-1484

18. Levy O, Martinowitz U, Oran A, Tauber C, Horoszowski H (1999) The use of fibrin tissue adhesive to reduce blood loss and the need for blood transfusion after total knee arthroplasty: a prospective, randomized, multicenter study. J Bone Joint Surg 81:1580-1588

19. Maloney WJ (2002) The stiff total knee arthroplasty: evaluation and management. J Arthroplasty 17(4 suppl1):71-73

20. Margolis DJ, Kantor J, Santanna J, Strom BL, Berlin JA (2001) Effectiveness of platelet releasate for the treatment of diabetic neuropathic foot ulcers. Diabetes Care 24:483-488

21. Marx RE, Carlson ER, Eichstaedt RM et al (1998) Plateletrich-plasma: growth factor enhancement for bone grafts. Oral Surg Oral Med Oral Pathol Oral Radiol Endod 85:638646

22. Mattingly PC, Bentley G, Cohen ML et al (1977) Preliminary experience with the geomedic total knee replacement. Rheumatol Rehabil 16:241-247

23. Nicholls DW, Dorr LD (1990) Revision surgery for stiff total knee arthroplasty. J Arthroplasty 5(suppl):S73-S77

24. Okuyama N, Wang CY, Rose EA et al (1999) Reduction of retrosternal and pericardial adhesions with rapidly resorbable polymer films. Ann Thorac Surg 68:913-918

25. Oz MC, Jeevanandam V, Smith CR et al (1992) Autologous fibrin glue from intraoperatively collected platelet-rich plasma. Ann Thorac Surg 53:530-531

26. Papagelopoulos PJ, Sim FH (1997) Limited range of motion after total knee arthroplasty: etiology, treatment, and prognosis. Orthopedics 20:1061-1067

27. Pham CT (2006) Neutrophil serine proteases: specific regulators of inflammation. Nat Rev Immunol 6:541-550

28. Ries MD, Badalamente M (2000) Arthrofibrosis after total knee arthroplasty. Clin Orthop Relat Res 380:177-183

29. Ritter MA, Campbell ED (1987) Effect of range of motion on the success of a total knee arthroplasty. J Arthroplasty 2:95-97

30. Schlag G, Redl H (1988) Fibrin sealant in orthopaedic surgery. Clin Orthop 227:269-285

31. Schliephake H (2002) Bone growth factors in maxillofacial skeletal reconstruction. Int J Oral Maxillofac Surg 31:469-484

32. Scranton PE Jr (2001) Management of knee pain and stiffness after total knee arthroplasty. J Arthroplasty $16: 428-435$

33. Sheppard BB, De Virgillio C, Bleiweis M, Milliken JC, Robertson JM (1993) Inhibition of intra-abdominal adhesions: fibrin glue in a long term model. Am Surg 59:786-790

34. Slater M, Patava J, Kingham K, Mason RS (1995) Involvement of platelets in stimulating osteogenic activity. J Orthop Res 13:655-663

35. Takeuchi H, Toyonari Y, Mitsuhashi N, Kuwabara Y (1997) Effects of fibrin glue on post surgical adhesions after uterine or ovarian surgery in rats. J Obstet Gynaecol Res 23:479-484 
36. Tawes RL, Sydorak GR, DuVall TB (1994) Autologous fibrin glue: the last step in operative hemostasis. Am J Surg 168:120-122

37. Thiede MA, Smock SL, Petersen DN, Grasser WA, Nishimoto SK, Thompson DD (1993) Production of osteocalcin by platelets: a potentially important link of platelet action in bone turnover. J Bone Miner Res 8:S147-S151

38. Toosie K, Gallego K, Stabile BE et al (2000) Fibrin glue reduces intra abdominal adhesions to synthetic mesh in a rat ventral hernia model. Am Surg 66:41-45

39. de Virgillio C, Elbassir M, Hidalgo (1999) Fibrin glue reduces the severity of intra-abdominal adhesions in a rat model. Am J Surg 178:577-580
40. Wang GJ, Hungerford DS, Savory CG et al (2001) Use of fibrin sealant to reduce bloody drainage and haemoglobin loss after total knee arthroplasty. J Bone Joint Surg Am 83:1503-1505

41. Weis AP, Krackow KA (1993) Persistant wound drainage after primary total knee arthroplasty. J Arthroplasty 8:285-289

42. Yamada Y, Ueda M, Naiki T, Takahashi M, Hata K, Nagasaka T (2004) Autogenous injectable bone for regeneration with mesenchymal stem cells and platelet rich plasma; tissue-engineered bone regeneration. Tissue Eng 10:955-964 